\title{
Signature of oscillations in coronal bright points
}

\author{
I. Ugarte-Urra ${ }^{1}$, J. G. Doyle ${ }^{1}$, M. S. Madjarska ${ }^{1,2}$, and E. O'Shea ${ }^{3}$ \\ 1 Armagh Observatory, College Hill, Armagh BT61 9DG, N. Ireland \\ 2 Mullard Space Science Laboratory, University College London, Holmbury St. Mary, Dorking, Surrey RH5 6NT, UK \\ ${ }^{3}$ Instituto de Astrofísica de Canarias, C/ vía Láctea s/n, 38200 La Laguna, Tenerife, The Canary Islands, Spain
}

Received 12 November 2003 / Accepted 23 January 2004

\begin{abstract}
A detailed study of two consecutive bright points observed simultaneously with the Coronal Diagnostic Spectrometer (CDS), the Extreme ultraviolet Imaging Telescope (EIT) and the Michelson Doppler Imager (MDI) onboard the Solar and Heliospheric Observatory (SOHO) is presented. The analysis of the evolution of the photospheric magnetic features and their coronal counterpart shows that there is a linear dependence between the EIT Fe XII $195 \AA$ flux and the total magnetic flux of the photospheric bipolarity. The appearance of the coronal emission is associated with the emergence of new magnetic flux and the disappearance of coronal emission is associated with the cancellation of one of the polarities. In one of the cases the disappearance takes place $\sim 3-4 \mathrm{~h}$ before the full cancellation of the weakest polarity.

The spectral data obtained with CDS show that one of the bright points experienced short time variations in the flux on a time scale of 420-650 s, correlated in the transition region lines (O V $629.73 \AA$ and O III $599.60 \AA$ ) and also the He I $584.34 \AA$ line. The coronal line (Mg IX $368.07 \AA$ ) undergoes changes as well, but on a longer scale. The wavelet analysis of the temporal series reveals that many of these events appear in a random fashion and sometimes after periods of quietness. However, we have found two cases of an oscillatory behaviour. A sub-section of the $\mathrm{O} v$ temporal series of the second bright point shows a damped oscillation of five cycles peaking in the wavelet spectrum at $546 \mathrm{~s}$, but showing in the latter few cycles a lengthening of that period. The period compares well with that detected in the S VI $933.40 \AA$ Ascillations seen in another bright point observed with the Solar Ultraviolet Measurements of Emitted Radiation (SUMER) spectrometer, which has a period of $491 \mathrm{~s}$. The derived electron density in the transition region was $3 \times 10^{10} \mathrm{~cm}^{-3}$ with some small variability, while the coronal electron density was $5 \times 10^{8} \mathrm{~cm}^{-3}$.
\end{abstract}

Key words. Sun: oscillations - Sun: corona - Sun: transition region - Sun: UV radiation - Sun: magnetic fields

\section{Introduction}

Coronal EUV/X-ray bright points (hereafter BPs) are small (20-30 Mm) coronal features of enhanced emission most easily observed in the quiet Sun and coronal hole regions. They were first observed and studied in soft X-ray rocket images (Vaiana et al. 1970; Nolte et al. 1979) where they present an average lifetime of $\sim 8 \mathrm{~h}$ (Golub et al. 1974). However, they have also been observed in radio (Marsh et al. 1980) and EUV (Habbal \& Withbroe 1981) where the emission has an average lifetime of $\sim 20 \mathrm{~h}$ (Zhang et al. 2001). The appearance and hence their description is subject to the spatial resolution of the instrument, so they are commonly presented as diffuse clouds with a central bright core of $\sim 10 \mathrm{Mm}$ as seen in Skylab X-rays (Golub et al. 1974) or SOHO/EIT extreme-ultraviolet images. High spatial resolution images $\left(1^{\prime \prime}-2^{\prime \prime}\right)$ have shown that BPs consist of several miniature loops (Sheeley \& Golub 1979) and have a

Send offprint requests to: I. Ugarte-Urra, e-mail: iuu@star.arm.ac.uk morphology similar to larger scale coronal structures (Frank \& Slater 2002).

BPs are associated with photospheric bipolar magnetic features (Krieger et al. 1971), with up to $2 / 3$ of them being associated with chance encounter and cancellation of pre-existing magnetic features rather than the emergence of new magnetic flux (Harvey 1985, 1993; Webb et al. 1993; Longcope et al. 2001). This process normally takes place at the network boundaries of super-granular cells (Egamberdiev 1983; Habbal et al. 1990; Madjarska et al. 2003).

One of the main characteristics of BPs is their intensity variability, as several studies in EUV spectral lines have shown. Sheeley \& Golub (1979) found that the constituent loops could evolve on a time scale of $\sim 6 \mathrm{~min}$. Habbal \& Withbroe (1981) and Habbal et al. (1990) using the Harvard experiment aboard Skylab showed that they exhibit large variations in the emission of chromospheric, transition region and coronal lines, and no regular periodicity or obvious correlation between the different temperatures was found. The time scales of these variations were as short as the temporal resolution of the observations 
(5.5 min). Similar variations were found in X-rays (Nolte et al. 1979). More recently, Madjarska et al. (2003) using SUMER observations with a temporal resolution of $50 \mathrm{~s}$, have confirmed the presence within a BP of small-scale transient brightenings of the order of minutes. Nevertheless, concerning BPs, very little has been done up to now to exploit the capabilities of the CDS and SUMER spectrometers onboard SOHO. Most of the studies have used EIT and MDI (also the Transition Region And Coronal Explorer, TRACE) and have focused on trying to understand the relationship between the BP coronal emission and its magnetic counterpart (see for example Preś \& Phillips 1999; Zhang et al. 2001; Longcope et al. 2001; Brown et al. 2001). Our intention in this paper is to fill part of this gap by studying the intensity variations of two BPs in different spectral lines with different formation temperatures. We also present the first wavelet analysis for BPs with the intention of checking the apparent lack of periodicity found so far.

\section{Observations}

All along this paper we are going to refer to two consecutive BPs observed in a coronal hole in November 2002. BP1 observed with EIT and MDI and BP2 observed as well with CDS.

\section{1. $C D S$}

On 7 November 2002, between 16:00 UT and 23:30 UT, and pointing at BP2 at coordinates $\left(-107^{\prime \prime},+355^{\prime \prime}\right)$, two studies representing temporal series of 81 and $30 \mathrm{~s}$ exposure time were run with the Normal Incidence Spectrometer NIS/CDS (Harrison et al. 1995) using the 4 " slit and scan mirror tracking. Telemetry restrictions only allow extraction of selected spectral windows from the two NIS detectors. We selected two wide windows (333.9-372.6 $\AA$, 567.5-631.3 $\AA$ ) for the first study, in order to better remove the continuum contribution. In the second study, we used four narrow windows centered at He I $584.34 \AA$, O III $599.60 \AA$, O v $629.73 \AA$ and $\mathrm{Mg}$ IX $368.07 \AA$. As a complement to the temporal series, a $60^{\prime \prime} \times 240^{\prime \prime}$ context image was obtained in three lines, He I $584.34 \AA$, O v $629.73 \AA$ and Mg IX $368.07 \AA$, alternating between the two studies.

The standard reduction was applied to the CDS data correcting for bias, flat-field, cosmic rays, and instrumental effects such as horizontal shifts due to the rotation of the scan mirror and rotation and tilt in the spectrum due to the misalignment between grating and detector, and grating and slit.

The small size and short lifetime of BPs added to the accuracy of the pointing, a few arcseconds, the small field of view and the fact that the pointing has to be given a few hours before the observing run, makes the choice of the target and the pointing an important issue in the observations of these features. For these reasons, "last minute pointing" ( $\sim 3 \mathrm{~h}$ before observing) was required, using as a reference the latest EIT images. The evolution, brightness and location of the BPs visible in the previous hours were inspected to find the appropriate target, i.e. a newly formed, bright and isolated (from active regions) BP that would hopefully remain visible until the end of the observing time.

\subsection{Context images and magnetograms}

As a context, we have EIT (Delaboudiniere et al. 1995) images in Fe XII $195 \AA$ and MDI (Scherrer et al. 1995) fulldisk magnetograms $\left(1.96^{\prime \prime} / \mathrm{pixel}\right)$, which cover with non regular cadences (6-90 min for the coronal imager and 1-90 min for MDI) the lifetime of the two BPs. In addition, we have one $1024 \times 1024$ pixel TRACE Fe IX/X $171 \AA$ image showing the fine structure of BP2 on November 8 at 00:34 UT.

In Fig. 1 the location of BP2 is highlighted with an arrow in a full-disk EIT $195 \AA$ image, with a close-up and a comparison with the TRACE image. It can be clearly seen that while in EIT $\left(2.6^{\prime \prime} / \mathrm{pixel}\right)$ the BP appears as a diffuse bright region with a brighter central core, the TRACE $\left(0.5^{\prime \prime} /\right.$ pixel $)$ image resolves it as an arcade of loops connecting opposite polarities. The alignment with MDI was done by cross-correlating EIT and TRACE images and matching the corrected TRACE coordinates with MDI.

MDI magnetograms were corrected for the solar differential rotation (Howard et al. 1990) and geometrical projection of the line-of-sight magnetic flux (Chae et al. 2001; Hagenaar 2001). Standard routines were used in the reduction of EIT and TRACE data (dark current subtraction, degridding, flat-fielding and comic rays replacements).

\section{Data analysis}

\subsection{BP identification}

The identification of a BP is usually done establishing a threshold value for its coronal emission. However, different criteria have been used in different works. For example, Habbal et al. (1990) used a factor of 2 higher flux than the surrounding quiet region in Mg X 625 while Zhang et al. (2001) used a $3 \sigma$ (root mean square) increase of the flux in an EIT $195 \AA$ quiet Sun area. Here, we have chosen the threshold based on the flux distribution (Brown et al. 2001). In Fig. 2 we plot the number of pixels vs. the $195 \AA$ flux for different EIT images of the same region with the BP emission still present (solid line) and when it has already disappeared (dashed line). It shows a tail in the distribution due only to the presence of the BP. We then choose a threshold value (dotted line) over which the enhanced coronal emission is considered as coming from a BP, which allows us to define its lifetime.

\subsection{CDS tracking}

Compensation for solar rotation can be done in two ways with CDS. Generally it is done by moving the rear legs that control the pointing of the instrument, however, this is not the recommended method when corrections of less than a few (35) arcsec are required (Pike 1997). It is more suitable to make use of the movement of the scan mirror, which re-points in steps of $2^{\prime \prime}$ in the E-W direction. There is a small difference between the correction applied by the mirror, in fixed steps, and the actual solar rotation. A cross-correlation of the two rasters at the beginning and end of the temporal sequences plus a comparison with the solar Y profiles of the slit shows that the 

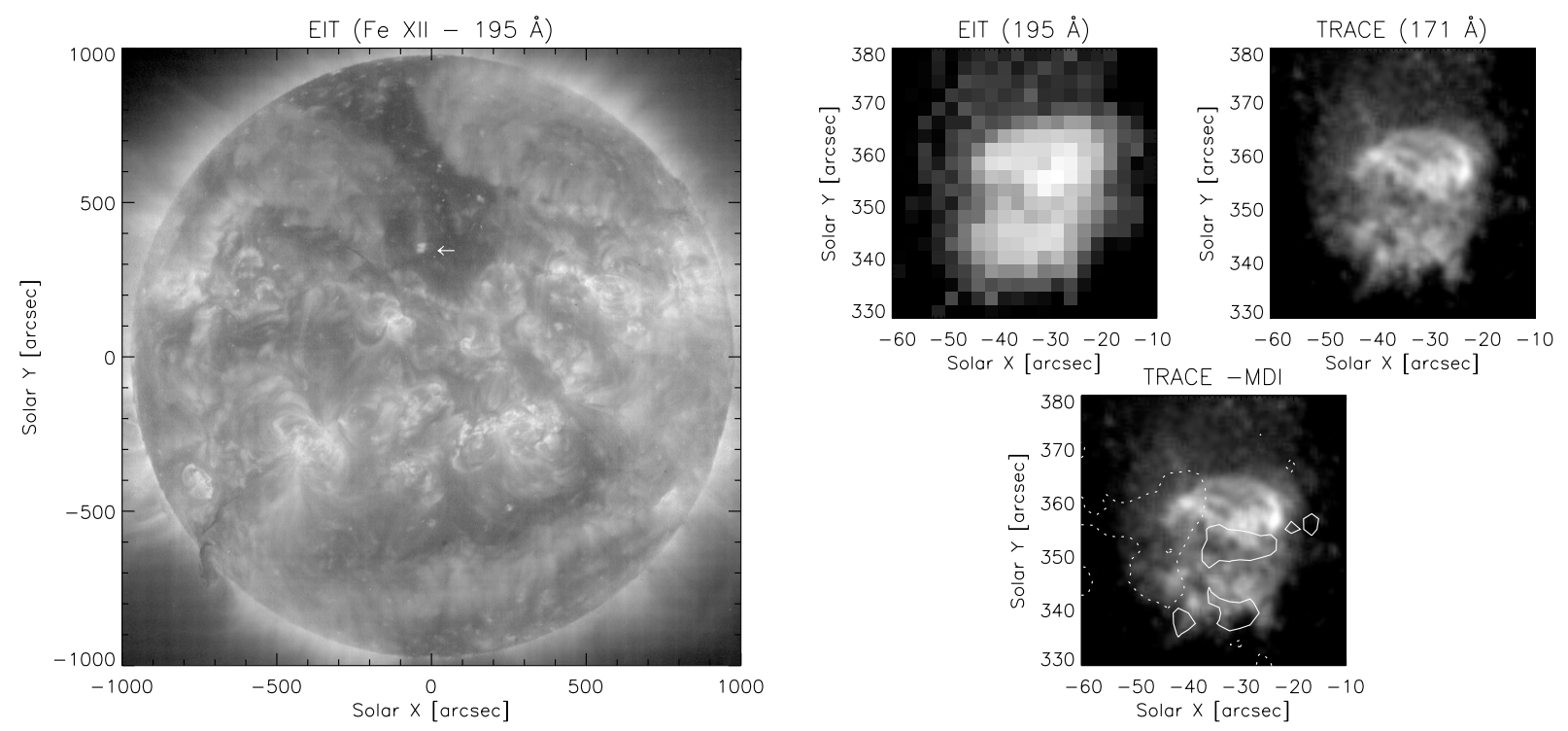

Fig. 1. BP2 location and semblance. Left image: context EIT Fe XII $195 \AA$ full disk image (8 November 2002, 00:36 UT) with an arrow highlighting the location of the BP. Top right images: comparison of a close-up of the feature in EIT and TRACE Fe IX/X $171 \AA$ taken 2 min earlier. Bottom right: magnetic field contours $( \pm 25 \mathrm{G}$, solid/dotted contours) overlaying the TRACE image. The arcade of loops resolved by TRACE connects opposite polarities.

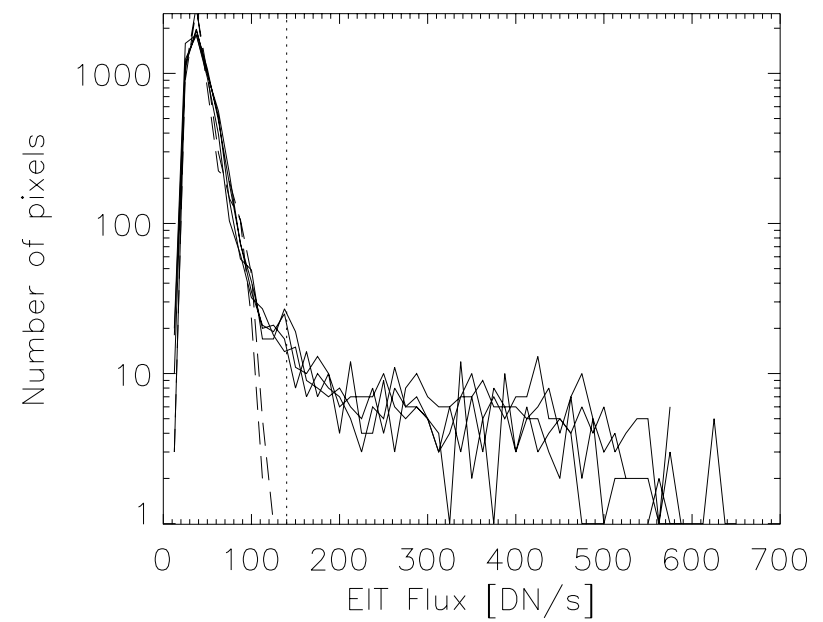

Fig. 2. Flux distribution for several EIT images of the same region, with no bright point (dashed lines) and with its presence (solid lines). The dotted line represents the threshold value chosen to define the BP.

cumulative effect after 120 min produces a drift of one pixel in the $\mathrm{E}-\mathrm{W}$ direction for our observing run.

\subsection{Spectral analysis}

It is well known in the CDS community that because of the loss of contact with SOHO in 1998, the performance of the instrument changed after the recovery. Most of these changes are well understood and can be dealt with, like the broadening and asymmetry found in the post-recovery spectral lines profiles of the NIS detector (Del Zanna et al. 2001, see Fig. B.2). These can be modeled with broadened Gaussian profiles (Thompson 1999). Nevertheless, extra care is needed in the subtraction of the continuum because the broadening produces a blending of the lines that hides the real continuum level in certain parts of the spectrum. This problem, which affects NIS 1 to a higher degree, can be avoided by extracting a wide spectral window, as the one shown in Fig. 3, where the continuum can be easily discriminated from the emission coming from blends and fitted with a first and a zero order polynomials. For the second study we selected narrow windows, but the only one that is registered in the NIS 1 detector is the Mg IX $368.07 \AA$ line and there is no difficulty in determining the continuum from the red wing. This line presents a blend with a Mg VII line at $367.68 \AA$, which was extracted by assuming that both lines have the same spectral width taking into account that they have a similar formation temperature. The other lines did not present any complex blends. Line fitting was carried out using the standard CDS package using broadened Gaussian profiles.

\subsection{Time series}

In order to get better signal to noise and taking into account that the spatial resolution of CDS is larger than the pixel size (Pauluhn et al. 1999), we summed the emission coming from several pixels along the slit. We selected the central pixels of the feature choosing in a quantitative approach those pixels with an intensity 3 times the $\sigma$ level of the emission in the raster, if available for that line, or along the slit otherwise. The binning was done then along 21-22 pixels in Mg IX, 16 pixels in $\mathrm{O} \mathrm{V}$ and $\mathrm{O}$ III, and 13-16 pixels in He I. In Fig. 4 we give an example of the procedure followed. On the left side, three rasters for three of the above lines are shown with the pixel location of the slit highlighted by a dashed line and a solid contour line accounting for the $3 \sigma$ level. On the right hand side, the plots correspond to the intensity profile along the dashed line on the raster. The dot-dashed line is the threshold level and the dotted lines delimit the interval of integration. 


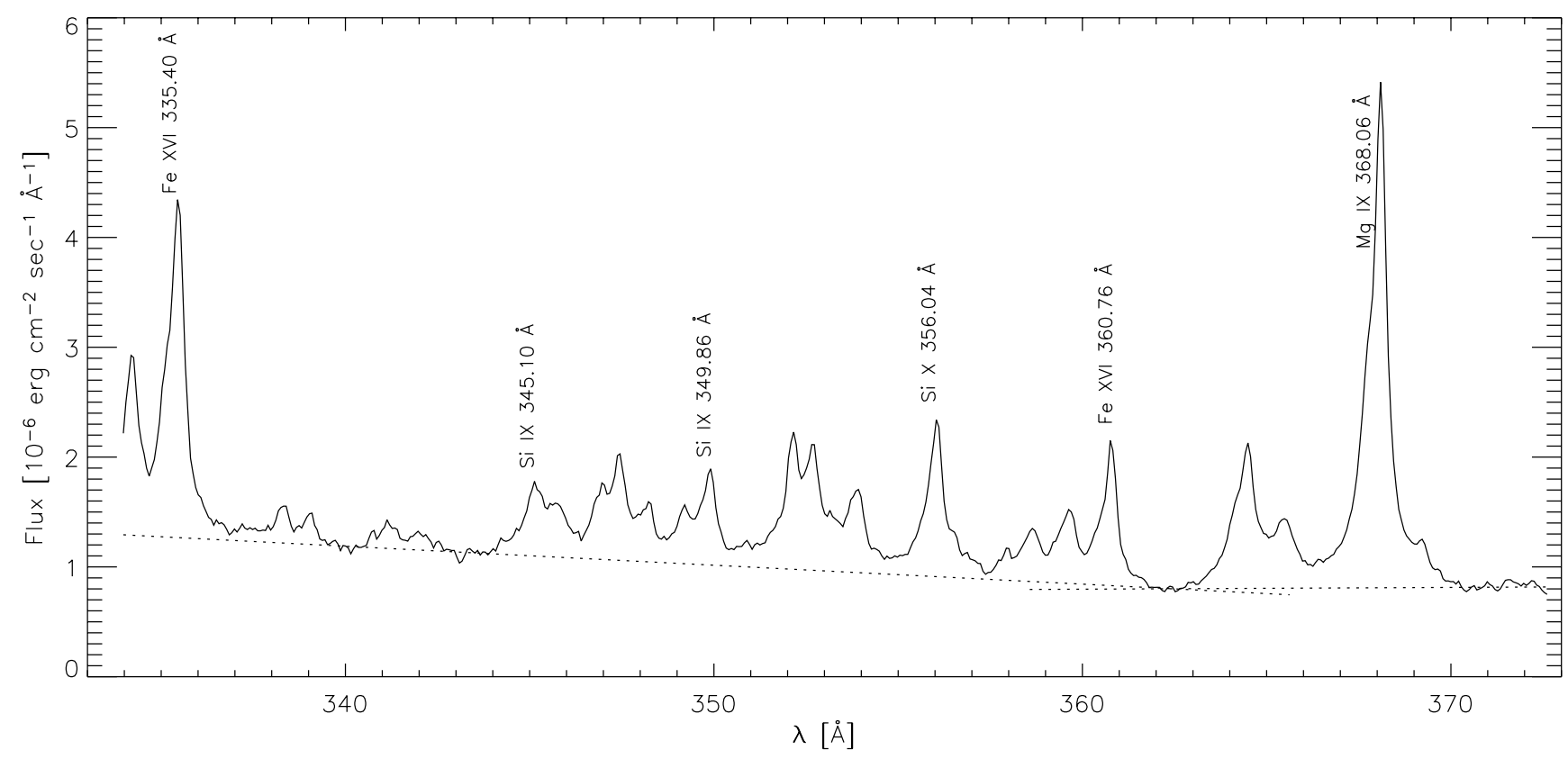

Fig. 3. Sample spectrum of the wide spectral window extracted from NIS 1 in one of the studies. The dotted lines represent the two fits used to subtract the continuum from the spectral lines. Some of the relevant ones are labeled.
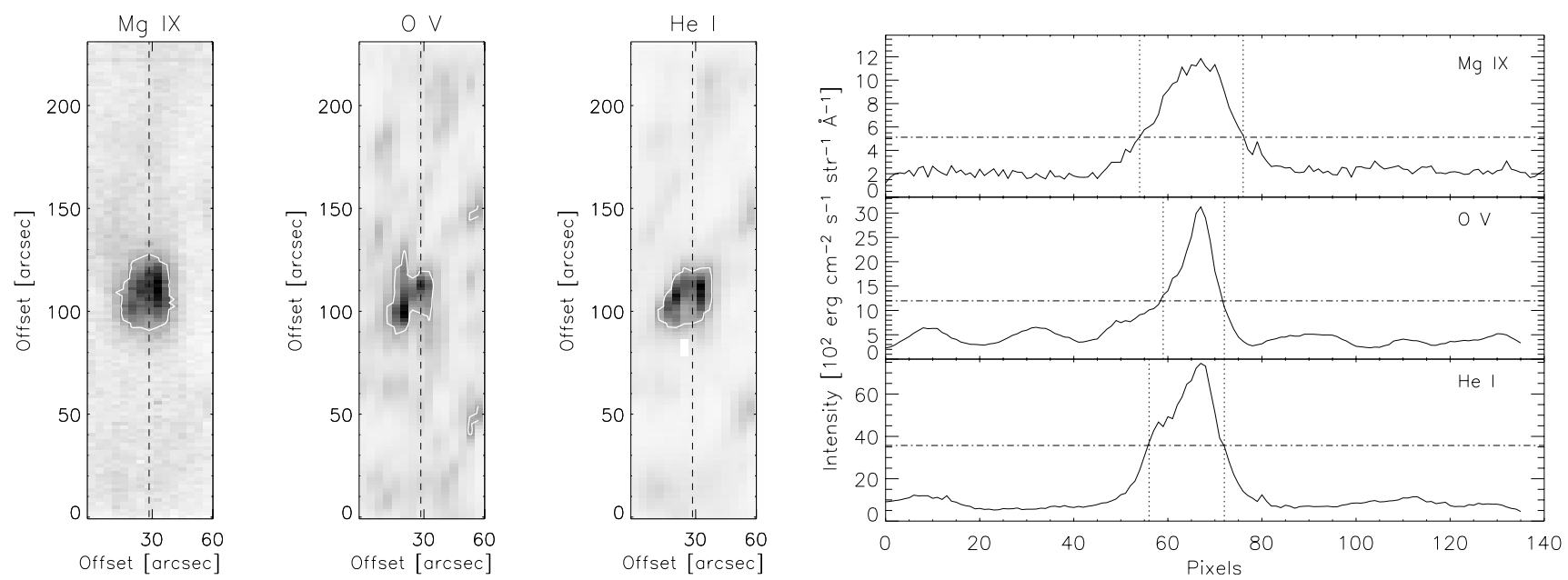

Fig. 4. In the left panels are Mg IX 368, O v 629 and He I 584 rasters for the BP of 7 November 2002 (BP2). The slit location for the spectral data is highlighted by a dashed line and a solid contour shows the $3 \sigma$ level. On the right hand panels, the plots correspond to the intensity profile along the dashed line in the raster. The dot-dashed line is the threshold level and the dotted lines delimit the interval of integration.

The appearance of BP2 in these rasters taken at the beginning of the first time series is representative of the whole dataset. The structure, although evolving and showing variations in intensity (to be discussed later), keeps approximately the same size and configuration along the whole sequence in the three lines, with the size in the transition region lines always slightly smaller than in the coronal line. This last statement fits the description given by Gallagher et al. (1998) for several very bright network features in their study of the properties of the quiet Sun EUV network.

The result of this binning are the temporal series to be discussed later. The average cadence between observations is $94 \mathrm{~s}$ for the run with a wide spectral window and $36 \mathrm{~s}$. for the two runs with narrow windows. The number of exposures $(80$ and 200) makes the total observing time $\sim 2 \mathrm{~h}$ for each of them.

\subsection{Wavelet analysis}

The wavelet analysis is now a common technique used to analyze non-stationary temporal signals. It has the advantage with respect to the fast Fourier transform that it can localize frequencies in time, making it suitable for the study of temporal series where different frequencies and different time localizations might be expected. A comparison of results obtained using both techniques can be seen for example in Lau \& Weng (1995) and Fludra (2001).

Software and definitions provided by Torrence \& Compo (1998) have been used in our study. The wavelet analysis consists of a convolution of the time series with a wavelet function resulting in a power spectrum, a two dimensional (time and frequency) transform of the temporal series. We chose the 
Morlet wavelet function. As in Fourier analysis, the highest values of the power spectrum correspond to the relevant frequencies present in the signal, although in this case with the time location also specified. This analysis suffers from edge effects at the limits of the time series. The region of the power spectrum where these effects are important is called the cone of influence (COI) and the results lying in this region should be disregarded.

A crucial part of the analysis is to find the significance levels in the power spectrum which tell us which are the real and relevant frequencies of the signal. We used a Monte Carlo or randomization method to estimate these levels (O'Shea et al. 2001). Randomization methods have the advantage that no assumption about a noise model is needed. It just assumes that if there is no periodicity in the time series then the intensity values are independent of the time of occurrence: any order of the intensity values in the time series would be as likely as any other one. The test then consists of permuting $n$ ! times the temporal series, with $n$ being the number of observations. Due to computational and time constraints (O'Shea et al. 2001) we restricted the calculations to a sample of $n=200$ random permutations. One then obtains the power spectrum for each of them and compares the peak values with the ones obtained from the data. The level of probability shown in our results is obtained from the proportion of times that the random peaks are larger than the peaks of the observed time series. We did that for the two highest maximums (hereafter 1st and 2nd maximum) of the power spectrum.

\section{Results}

\subsection{Lifetime and evolution}

According to the criteria of identification described earlier, the BP studied with CDS (BP2) appeared in the EIT images on November 7 at 04:48 UT and disappeared at 19:26 UT on the 8th, which means that it had a lifetime of $\sim 38 \mathrm{~h}$ in the $195 \AA$ emission. From the magnetic field point of view the polarities were present much earlier. In fact, another bright point (BP1) was visible before this one at the same location. It appeared on November 4 at 17:36 UT with the emergence of a positive polarity in an area of dominant negative polarity. After several hours of interaction, the positive polarity canceled almost totally with the subsequent fade of the coronal emission (November 6, between 20:48 UT and 22:24 UT), leaving a pair of small positive fragments $\sim 10-13 \mathrm{Mm}$ apart from the negative one. After an interval of five hours, new negative flux emerged at $\sim 6 \mathrm{Mm}$ distance from the positive ones and BP2 appeared. During its lifetime, BP2 grew in area and intensity lying always between the two polarities or covering the whole bipolarity. The negative polarity being the dominant one with several of the surrounding negative features joining in during the process. The positive fragments, meanwhile, were gradually and totally canceled by the negative ones until BP2 faded. This dissipation occurred $\sim 3-4$ hours before the full cancellation of the polarity, which took place some time between 22:24 UT and 00:00 UT on the 9th.

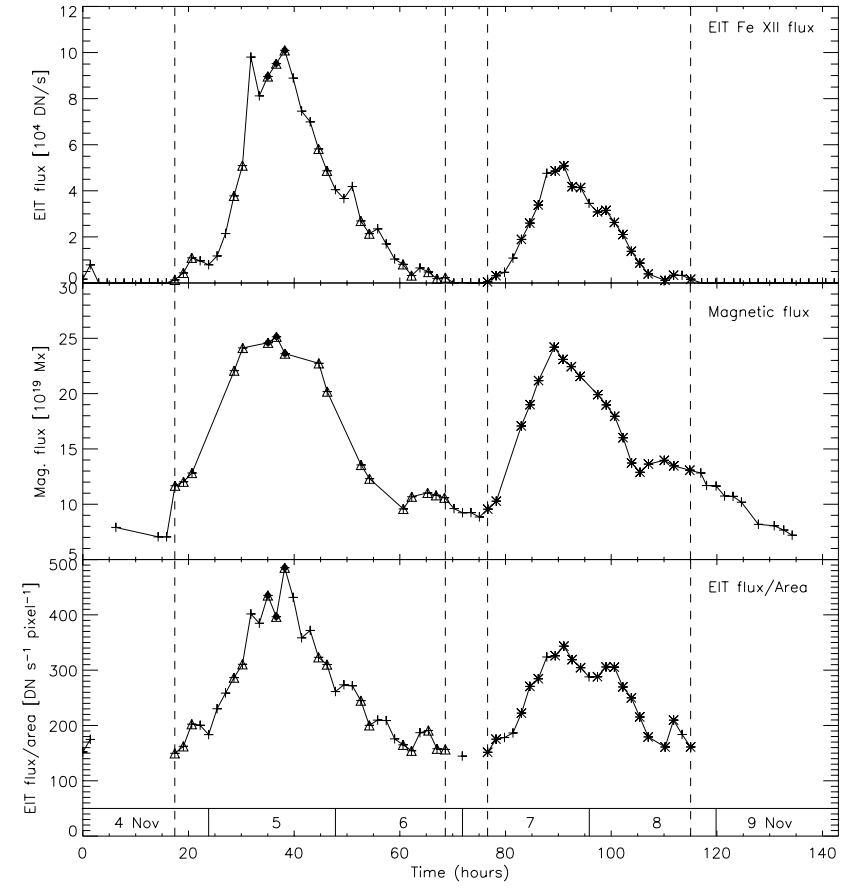

Fig. 5. Evolution in time of the EIT flux, magnetic flux and EIT flux per unit area (pixel) of the two consecutive BPs observed at the same location. Dashed lines delimit their lifetime. The observed data-points are represented by plus symbols, triangles represent times with simultaneous EIT and MDI data for BP1 and stars for BP2. The black dots are three points to be referred to in Fig. 6 . The starting time is November 4, 2002, 00:00 UT.

In Fig. 5 the evolution in time of the EIT flux and magnetic flux of the two bright points can be seen. The starting time is November 4 at 00:00 UT. The EIT flux accounts for the integrated emission of the pixels with flux over the identification threshold. The total unsigned magnetic flux $(\phi=$ $\left.\int B_{l} \mathrm{~d} S\right)$ was calculated integrating the line of sight magnetic field strength $\left(B_{1}\right)$ measured on the MDI magnetograms (300 s/exposure) over a $60^{\prime \prime}$ square area below the coronal emission region using a cutoff value of \pm 25 Gauss. The two top panels show the important role that the magnetic field plays in the evolution of BPs, as other authors have already shown (Preś \& Phillips 1999; Madjarska et al. 2003). However, taking into account that the area evolves following a similar trend to the coronal emission, we should look at the evolution of flux per unit area (pixel) at the bottom graph. We see that the behaviour of the EIT flux is not only due to the increase/decrease of the BP area, but also to the intrinsic evolution of the feature (Habbal \& Withbroe 1981), which has a higher intrinsic emission when the magnetic flux is at its maximum.

The dependence of the EIT flux on the total magnetic flux can also be inferred from Fig. 6, where the symbols have the same meaning as in Fig. 5. There is a linear dependence between these two quantities, excluding the three black dots. They represent the points with maximum flux and area for BP1 and it seems that the high increase in intensity at these times is not related to an increase of magnetic flux, which remains almost constant. It is interesting to note that the steepness of the linear fits to the data points of both days (dashed line: BP1; 


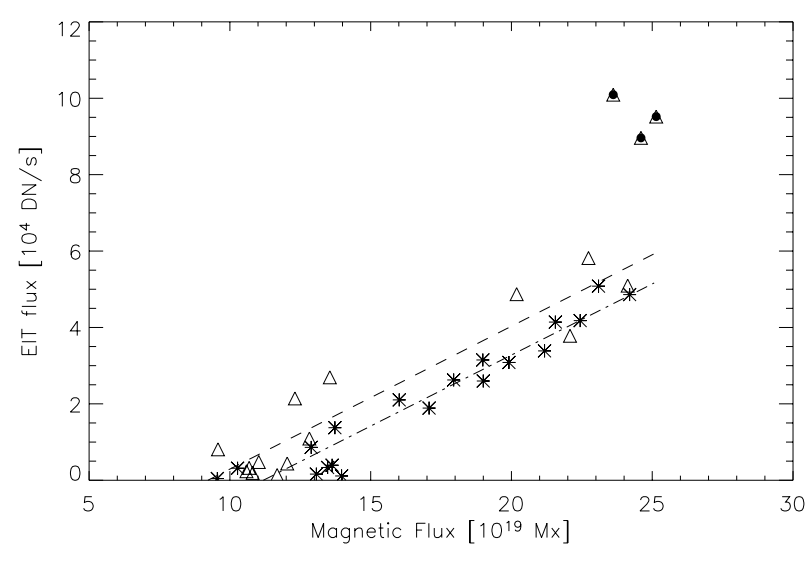

Fig. 6. Relation between the EIT Fe XII flux and the magnetic flux. Same symbols as in Fig. 5 are used. The dashed line is the linear fit to the BP1 points (triangles) excluding the three black dots, and the dot-dashed line is the fit to the BP2 points (stars).

dot-dashed: BP2) is basically the same, which suggests that for similar magnetic strengths BPs evolve similarly in intensity. The fit is subjected to the uncertainty in the method used to calculate the magnetic flux. However, introducing a time dependent area of integration does not change the results and conclusions, but marginally the steepness of the fit.

We have also checked the proposed dependence of the lifetime with the maximum area suggested by Golub et al. (1974): $A_{\text {max }}=2.5 \times 10^{7} \tau\left[\mathrm{km}^{2}\right]$, where $\tau$ is the lifetime in hours. The predicted maximum area values are around 3 times larger than the observed ones. BP1 has a lifetime of around $51 \mathrm{~h}$ and maximum area of around $4.8 \times 10^{8} \mathrm{~km}^{2}$, and BP2 $38 \mathrm{~h}$ and $3 \times 10^{8} \mathrm{~km}^{2}$, while the prediction gives $12 \times 10^{8}$ and $9.5 \times 10^{8} \mathrm{~km}^{2}$, respectively.

\subsection{Time series}

If we focus now on the CDS study, the temporal response of the BP (BP2), as seen from different lines corresponding to different temperatures of the solar atmosphere, is shown in Fig. 7. The time series for each of the lines consist of three consecutive sections: firstly the sequence run with longer exposure time (81 s/exposure), followed by two sequences of $30 \mathrm{~s} /$ exposure. The two gaps in between the three time series were covered by context rasters. Other gaps are just missing data. The vertical dotted lines account for the moments when the scan mirror was moved for tracking. The plots show that the BP experiences short time variations (order of minutes) in the flux. This manifestation is clear for the two transition region lines (O V $629 \AA$ and O III $599 \AA$ ) and slightly smoother for the He I 584 line. The coronal line (Mg IX $368 \AA$ ) undergoes changes too, but on a longer scale. The signal-to-noise is lower for this line, even though we binned more pixels, and the error bars are larger. However, it does not seem to hide these large fluctuations of the flux seen in the cooler lines. These fluctuations are not ubiquitous in time and can occur after a quiet period like the one seen in the second section of the time series, $2 \mathrm{~h} 27 \mathrm{~min}$ after the start of the series. The start time in the plot is November 7 at 16:20 UT.
The high activity seen in the transition region lines is highly correlated at different temperatures, as a glance to the two middle panels shows. There is a one to one relation for the bursty changes, and the general trend is certainly followed by the He I line, reproducing even some of the peaks. Nevertheless, the changes are less important with the maximum variation in the amplitude being $40 \%$ at the beginning of the series, with an average of $10-30 \%$ from troughs to crests. For O V, the variations are of the order of 30-60\%. Within the temporal resolution of our data, there is no noticeable time delay between the peaks at different temperatures.

\subsection{Wavelet analysis results}

It is clear from the present dataset that if we want to investigate if there is any periodicity in these transient intensity changes, we need to use a technique capable of detecting different periods or frequencies at different times. As discussed before, wavelet analysis can do this. We present now the analysis of two BPs: first the one described in previous sections and next a BP studied by Madjarska et al. (2003) with SUMER.

\subsubsection{Present dataset}

An example of the wavelet analysis carried out is shown in Fig. 8. The top panel shows the time evolution of the intensity in photon-events. It is, in fact, a detrended intensity, result of the subtraction to the original series of the linear fit to each of the intervals between tracking corrections (dashed lines in Fig. 7), as done by Fludra (2001). The power spectrum is shown in the middle panel, with the level of probability corresponding to the two higher maximums of the power for each time location in the bottom panel. The 1st maximum is represented by thick dots in the power spectrum plot and by a solid line in the probability one; the 2 nd by thin dots and by a dotted line. The global wavelet spectrum, an average in time of the power spectrum, lies on the right-hand side.

A summary of the results is presented in Table 1 for each of the three studies (s26195, s26197 and s26199) shown in Fig. 7. An analysis was done for each of the three lines: Mg IX $368 \AA$, O V $629 \AA$ and He I $584 \AA$. The periods obtained for the two highest maximums in the global wavelet spectrum are followed by the level of probability $(1-p)$, with $p$ being the proportion in the number of permutations where the maximum of the randomized power spectrum is higher than the maximum of the real power spectrum. It gives an indication of how reliable the results are. Only periods with a level of probability higher than $0.95(95 \%)$ are considered as possible candidates for a periodic signal in this paper. These values are the result of a comparison between the global wavelet spectra of real and random series. Values of 1.0 just indicate that the probability is between 99-100\% (O'Shea et al. 2001). We will refer to the power and probability plots for cases when time plays an important role. The relevant periods have been highlighted with bold font. COI in the comments means that the maximum in the power accounts for a signal which falls inside the cone of 


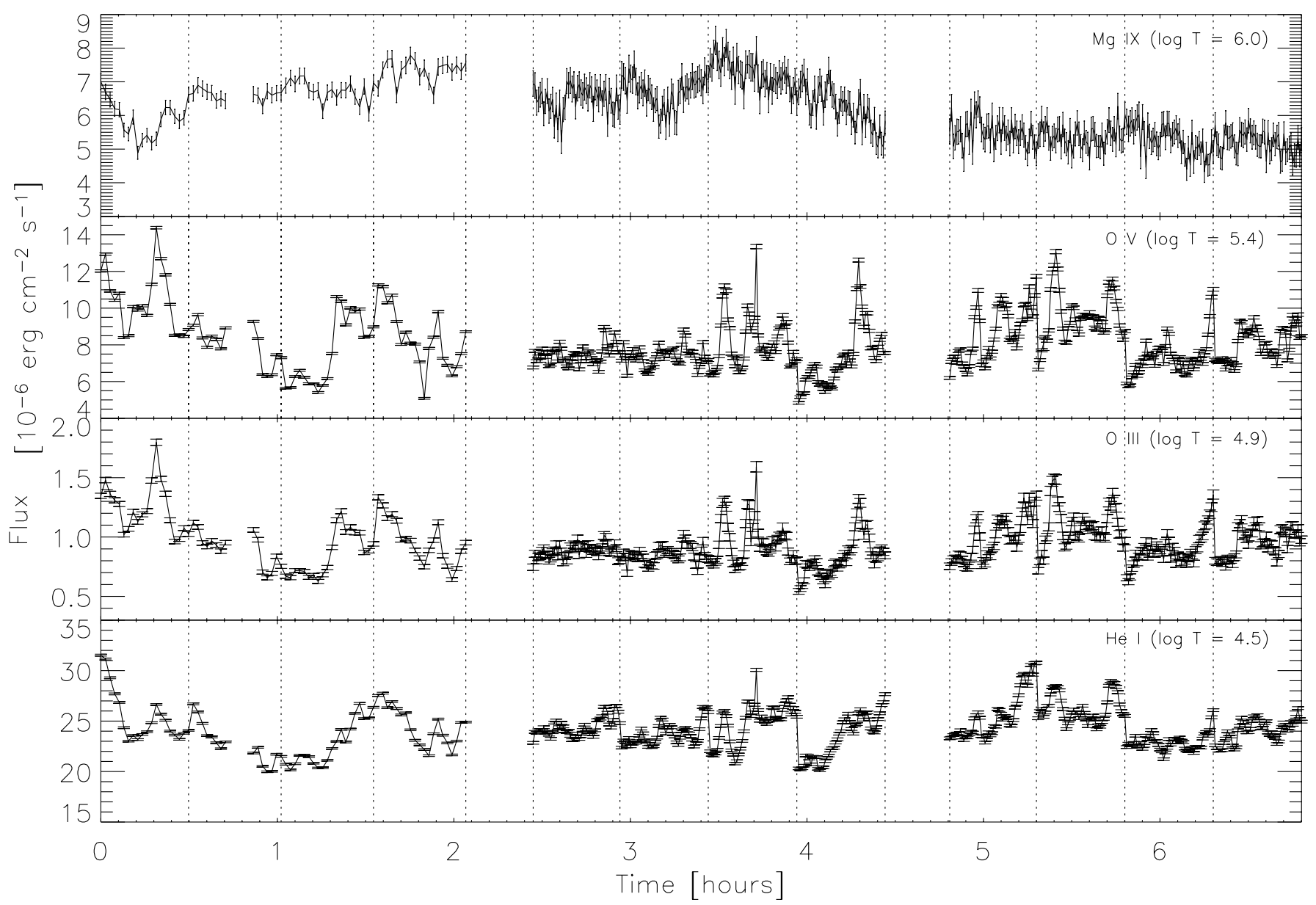

Fig. 7. Flux variations for BP2 as seen in Mg IX, O v, O III and He I. Start time is November 7 at 16:20 UT. The three sections correspond to studies s26195, s26197 and s26199. Gaps in between were covered by context rasters. Dotted lines signal when the tracking correction took place. The formation temperature of the spectral line is provided beside its identification.

Table 1. BP2 wavelet results. Columns: study number, spectral line used, maximum of the global wavelet power spectrum, period associated with the maximum, probability $(1-p)$, and comments to the results. COI: signal located inside the cone of influence; numbers: reference to the text. The relevant periods are in bold font.

\begin{tabular}{cccccc}
\hline \hline Study & Line & Max. & Period [s] & Prob. & Comments \\
\hline s26195 & Mg IX & 1st & 327 & 0.34 & \\
& & 2nd & 1698 & 0.05 & \\
& O v & 1st & $\mathbf{1 0 1 0}$ & 1.00 & $(1)$ \\
& & 2nd & 2020 & 0.91 & \\
& He I & 1st & 1852 & 1.00 & COI \\
& & 2nd & 1010 & 0.93 & COI \\
s26197 & Mg IX & 1st & 1300 & 0.99 & $(2)$ \\
& & 2nd & 709 & 0.25 & \\
& O v & 1st & $\mathbf{5 9 6}$ & 1.00 & $(3)$ \\
& & 2nd & 1003 & 0.98 & COI \\
& He I & 1st & $\mathbf{9 1 9}$ & 1.00 & $(4)$ \\
& & 2nd & $\mathbf{4 2 2}$ & 1.00 & $(5)$ \\
s26199 & Mg IX & 1st & 709 & 0.67 & \\
& & 2nd & 1417 & 0.46 & \\
& O v & 1st & $\mathbf{5 4 6}$ & 1.00 & $(6)$ \\
& & 2nd & $\mathbf{1 0 0 2}$ & 1.00 & $(7)$ \\
& He I & 1st & 1838 & 1.00 & COI \\
& & 2nd & $\mathbf{5 9 6}$ & 1.00 & $(8)$ \\
\hline
\end{tabular}

influence, so it is disregarded. Other comments are referred as numbers in the text.

The analysis of study s26195 in O v $629 \AA$ produces a peak in the power at $1010 \mathrm{~s}$ with the probability over the 0.95 level (1), identified in the time series with several peaks with a response time or duration close to the period. For He I 584, where the changes are smoother, the analysis only detects an initial peak inside the COI with a probability value of 0.93 . It is worth mentioning an isolated peak with a lifetime of $566 \mathrm{~s}$ experienced by $\mathrm{O} \mathrm{V}$ at the beginning of the time series (not given in Table 1).

Study s26197 shows a period of $1300 \mathrm{~s}$ with over $95 \%$ probability for the noisy Mg IX $368 \AA$ time series (2). An inspection of the time dependent plots shows that it is due to the modulation seen in Fig. 7, some $3 \mathrm{~h}$ into the observations. If we remove this low frequency with a 20-point running average, there are no relevant periods remaining in the data. The O v $629 \AA$ time series, however, show a very clear peak at around $596 \mathrm{~s}$ (3), see Fig. 8, which is associated with an interesting trend. Figure 9 is a section of the series, just after minute 60 from the start. There are three consecutive brightenings with a similar response time ranging between 546 and $596 \mathrm{~s}$, during a period where no tracking correction was necessary, followed after the correction by two more brightenings of decreasing intensity with the peak of 

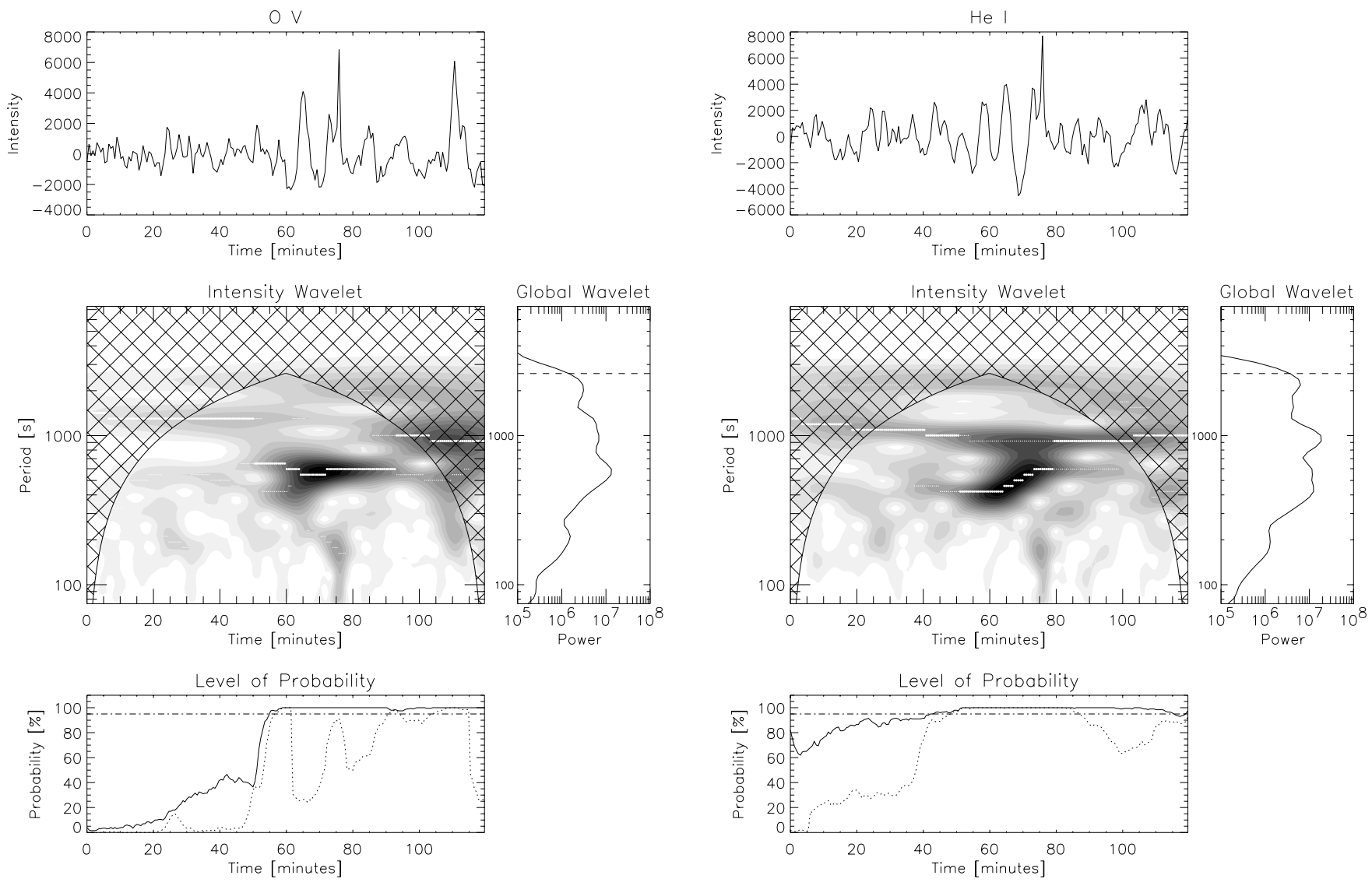

Fig. 8. BP2 wavelet results corresponding to study s26197 (middle series in Fig. 7) for O v $629.73 \AA$ A (left) and He I $584.34 \AA$ (right). Top: detrended intensity in photon-events; center: wavelet power spectrum and global wavelet spectrum; bottom: level of probability for the two highest peaks in the spectrum. 1st maximums: thick dots and solid line; 2nd maximums: thin dots and dotted line. The dash-dotted line corresponds to a probability level of 95\%. Start time is November 7, 2002 at 18:47 UT. The dashed line of the global wavelet plot represents the maximum period outside the COI.

the wavelet inside the COI. For a qualitative understanding of what clearly appears as an oscillatory behaviour we have overplotted an exponential damped sine function of amplitude

$A(t)=A_{0} \sin \left(\frac{2 \pi t}{P}-\phi_{0}\right) \exp \left(-\frac{t}{t_{\mathrm{d}}}\right)$

with the initial amplitude $A_{0}=4200$ photon-events, the period $P=546 \mathrm{~s}$ (obtained in the wavelet analysis), the phase $\phi_{0}=$ $7 \pi / 11$ and the exponential decay time $t_{\mathrm{d}}=1800 \mathrm{~s}$. The profile reproduces well the intensity behaviour, suggesting that there is a damped oscillation with a period that increases with the damping. A strong brightening very similar to the initial ones takes place just after (or even during) the weakest of the events. Nothing can be said from the wavelet because its maximum is inside the COI, but the duration, as seen in the light curve, is from minimum to minimum $\sim 560 \mathrm{~s}$, a typical response time of other events.

In He I $584 \AA$ there is no sign of the damped oscillation, however, the two first brightenings are also present with a maximum in the wavelet at similar values for the period (Fig. 8 right). Preceding them there are high power spectrum values at $422 \mathrm{~s}$ that produce the second maximum in the global wavelet (5), also seen for O V $629 \AA$ as a second maximum (thin dots) on the left plot. At 20:03 h UT, 76 min after the starting point of the two plots in Fig. 8, one of these brightenings experiences what seems like a flaring event with a $50 \%$ increase in the O v $629 \AA$ flux of one of the datapoints, also seen in Fig. 9. The same increase is found in the other transition region line, O III 599, and a 15\% increase in He I $584 \AA$. In the coronal line, although weaker, there is a jump of a $10 \%$ just visible over the error bars. The length of the event is given by the time resolution of the time series, i.e. $72 \mathrm{~s}$. An inspection of the unbinned data reveals that the event peaks in a single spatial pixel spreading to the neighbours probably due to the fact that the spatial resolution is larger than the pixel size (Pauluhn et al. 1999). Finally, a 919 s period seems to modulate the $\sim 500$ s peaks at the center of the series in He I during 3 complete cycles (4).

The last of the studies is s26199. As in the previous ones, the coronal line time series does not reveal any relevant frequency over the noise. (6) O V $629 \AA$ shows again a peak at $546 \mathrm{~s}$ and the time dependent plot shows different consecutive brightenings with periods varying between 459 and $596 \mathrm{~s}$ at the start of the series. In He I $584 \AA$, for the same brightenings, we found the maximum in the wavelet power for periods ranging from 459-649 s, with a maximum in the global wavelet 


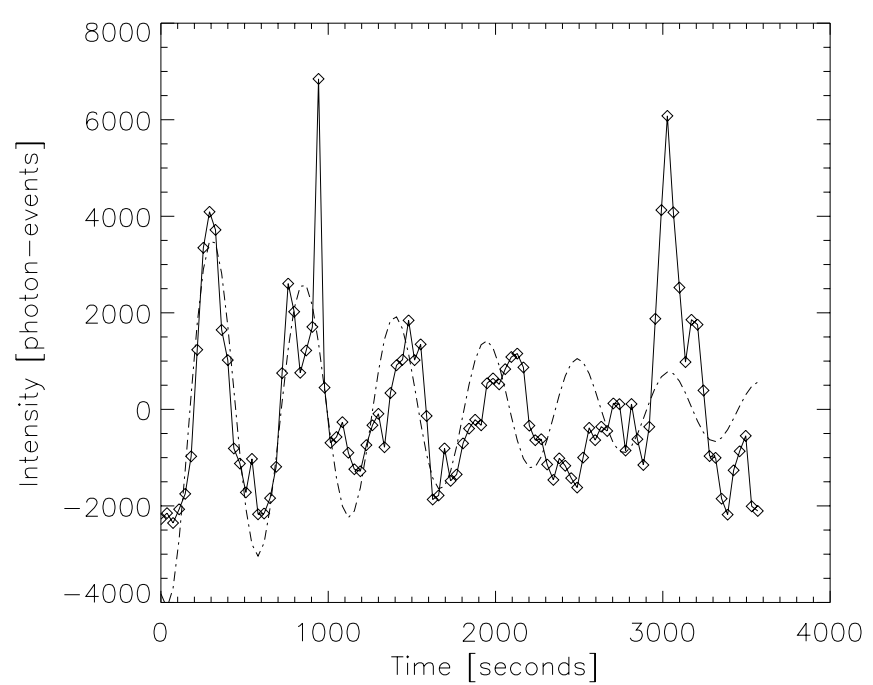

Fig. 9. Damped oscillation observed in the O v 629 time series, study s26197. Overplotted in dot-dashed line there is an exponential damped sine function with a period of $546 \mathrm{~s}$.

Table 2. Wavelet results for the BP observed with SUMER on October, 17, 1996. Columns: spectral line, maximum of the global wavelet spectrum, period associated with the maximum and level of probability.

\begin{tabular}{cccc}
\hline \hline Line & Maximum & Period [s] & Prob. \\
\hline S VI & 1st & $\mathbf{4 9 1}$ & 1.00 \\
& 2nd & $\mathbf{9 0 1}$ & 1.00 \\
\hline
\end{tabular}

of 596 (8). The table shows also a peak at $1002 \mathrm{~s}$ in $\mathrm{O} \mathrm{V}$ (7) that could modulate the center of the series.

\subsubsection{SUMER BP}

We have also inspected a BP observed on October 17, 1996 with several SOHO instruments, where a detailed study was done by Madjarska et al. (2003). We concentrate on the intensity changes observed in the S VI $933.40 \AA$ line obtained with the SUMER spectrometer and shown in Fig. 10 of that reference. The observation was done in the sit-and-stare mode, i.e. no tracking correction was applied. The time cadence of the series after a bin of 5 spectra (10 s/exposure) is $50 \mathrm{~s}$. In that figure the high intensity average values at the center of the time profile and the low ones at the wings are just a consequence of the BP moving outside the slit, entering and leaving. This trend was removed from the time series using a 30-point running average, and the result is plotted at the top of our Fig. 10. The result of the wavelet analysis is shown in Table 2 .

As explained in the cited paper, due to solar rotation the SUMER slit ( 1 ") covered a new region of the Sun after $\sim 390 \mathrm{~s}$, so that variations occurring during a period of 400-500 s can be seen as a temporal change in a small scale phenomenon. We have found four brightenings, after minute 40 from the start of the series, which have characteristic periods in the range of 451-491 s, which means that these are real temporal changes in the BP constituents. The oscillatory nature of these intensity changes can be seen in Fig. 11. A sinusoidal function with a
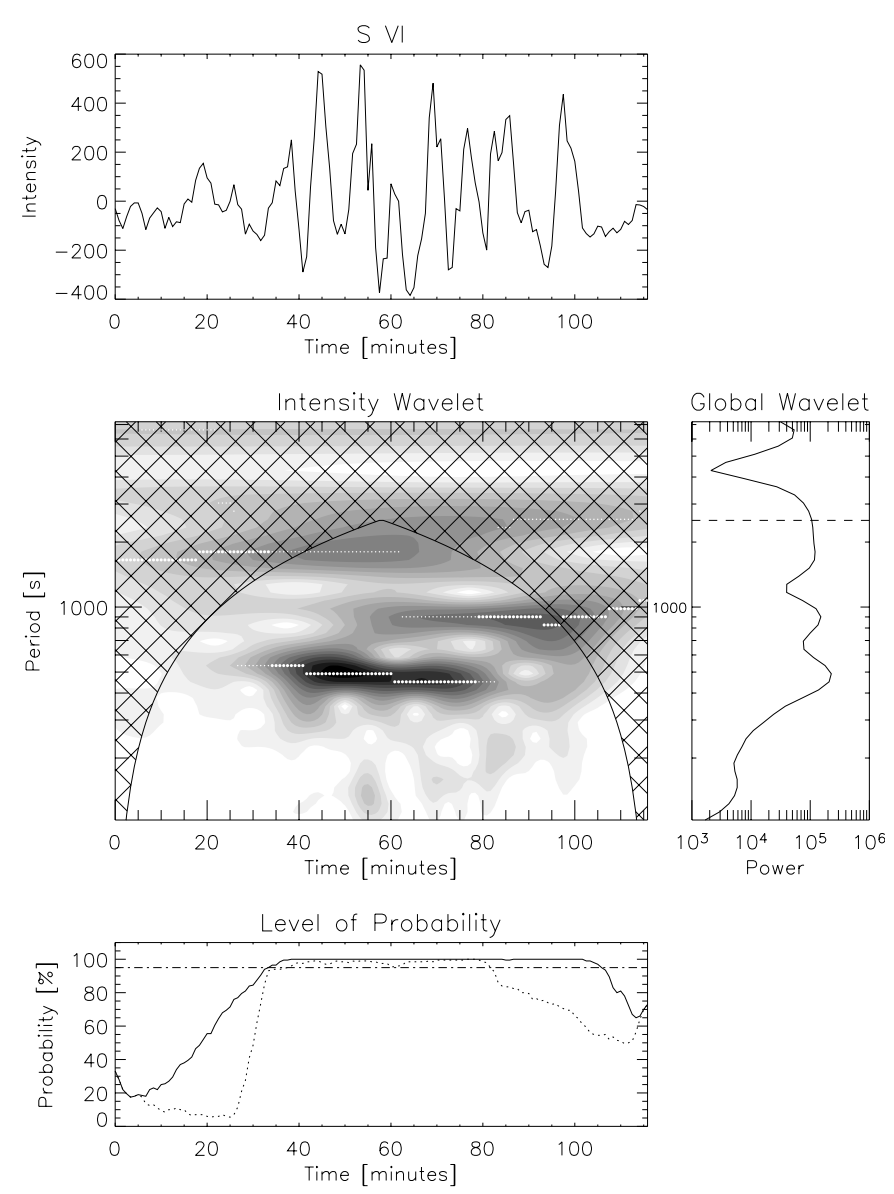

Fig. 10. Wavelet results for the SUMER BP. See caption in Fig. 8 for details. Units for the intensity are counts.

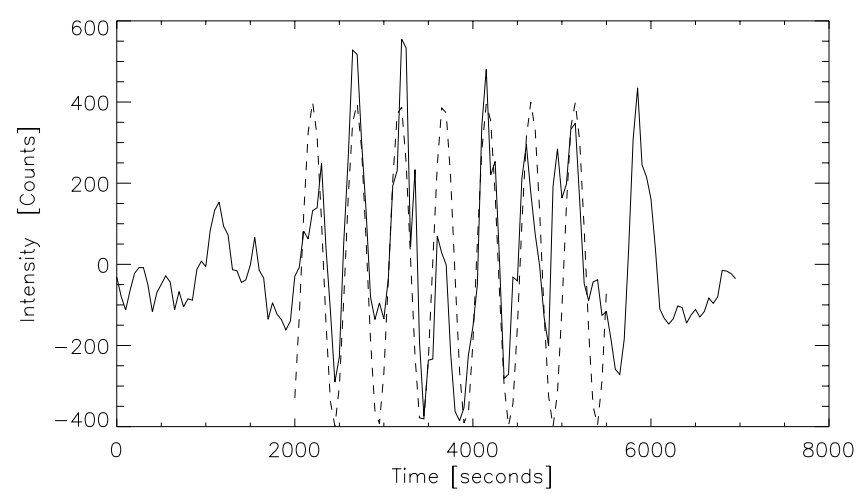

Fig. 11. S vI detrended intensity fluctuations (solid line) corresponding to the SUMER BP. Overplotted with a dashed line there is a $491 \mathrm{~s}$. period sinusoidal function.

period of $491 \mathrm{~s}$, as derived from the wavelet analysis, is overplotted on the detrended intensity fluctuations. It is clear from the comparison of the two curves that there is a regularity in the appearance of the brightenings. These "short" brightenings are followed by longer ones with a peak at $901 \mathrm{~s}$. 


\subsection{Electron density}

The temporal variation of the electron density $\left(N_{\mathrm{e}}\right)$ associated with the intensity changes was also investigated. The determination of $N_{\mathrm{e}}$ was done using the CHIANTI atomic database (Dere et al. 1997; Young et al. 2003) and the ionization equilibrium of Mazzotta et al. (1998). The identification of the lines and possible blends was obtained using CHIANTI, the list given in the quiet Sun EUV spectrum observed with CDS (Brooks et al. 1999) and the list given by Del Zanna (1999). Several density sensitive line ratios are present in the dataset including ratios of Si IX, Si X and Fe XII. However, despite our best efforts, the post-recovery broadening that affects the profiles of the lines, mainly in detector NIS 1, does not make the fitting procedure an easy task in the region where these lines are located, namely in the range 340-347 $\AA$ and $364 \AA$ in the case of a Fe XII line (see Fig. 3). Although the fitting can be done in certain cases, the uncertainties and assumptions involved do not allow us to have sufficient confidence to present a temporal variation. The Si IX ratio 349.86/345.10 gives an average value of $\sim 5 \times 10^{8} \mathrm{~cm}^{-3}$ consistent with the value presented by Del Zanna et al. (2003) for a BP in a coronal hole using a Si IX ratio. Line profiles from the second detector (NIS 2) presented a better prospect and the $\mathrm{O}$ IV density sensitive line ratio (625.8/608.4) was successfully determined. The $608.4 \AA$ line is part of a set of several lines including the second order Si XI $606.8 \AA$ and He II $607.6 \AA$ lines, and a blend of a Mg X line with an O IV one at $609.9 \AA$. The $608.4 \AA$ O IV line was fitted using the width obtained for $\mathrm{O} v$, which is close in temperature of formation. The $625.8 \AA$ line is located in the red wing of the $\mathrm{Mg}$ X $625.0 \AA$ and even though is not a very strong line it can be safely fitted.

As the BP is located in a coronal hole area, the background emission is not very high. However, it is still important to do a proper subtraction. For the $608.4 \AA$ line, we used a close-by region to the $\mathrm{BP}$, where no network emission is seen and which remains fairly constant. We avoided regions where bumps in the emission could be due to network oscillations. The background emission of the $625.8 \AA$ was too weak to be fitted by a Gaussian and could not be removed, so the $N_{\mathrm{e}}$ values presented here are an upper limit. If the background is not subtracted at all, the general behaviour (shown in Fig. 12 and discussed below) still remains the same, but the values are lower (as one would expect) by on average $30 \%$, which shows the importance of subtracting the background emission in order to get accurate $N_{\mathrm{e}}$ values.

Figure 12 shows the temporal changes of the flux for the two O IV lines. The start time is the same as in Fig. 7. The gap accounts for missing data and the vertical dotted lines serve just as reference. The fact that the time changes are the same for both lines is a good indication that the fitting and its assumptions are reliable. The plot at the bottom shows the temporal response of the electron density. Even though the errors, which come from the propagation of the intensity errors through the ratio, are large, from this plot we suggest that the intensity changes are probably the result of electron density changes in the transition region. Unfortunately, we do not have another
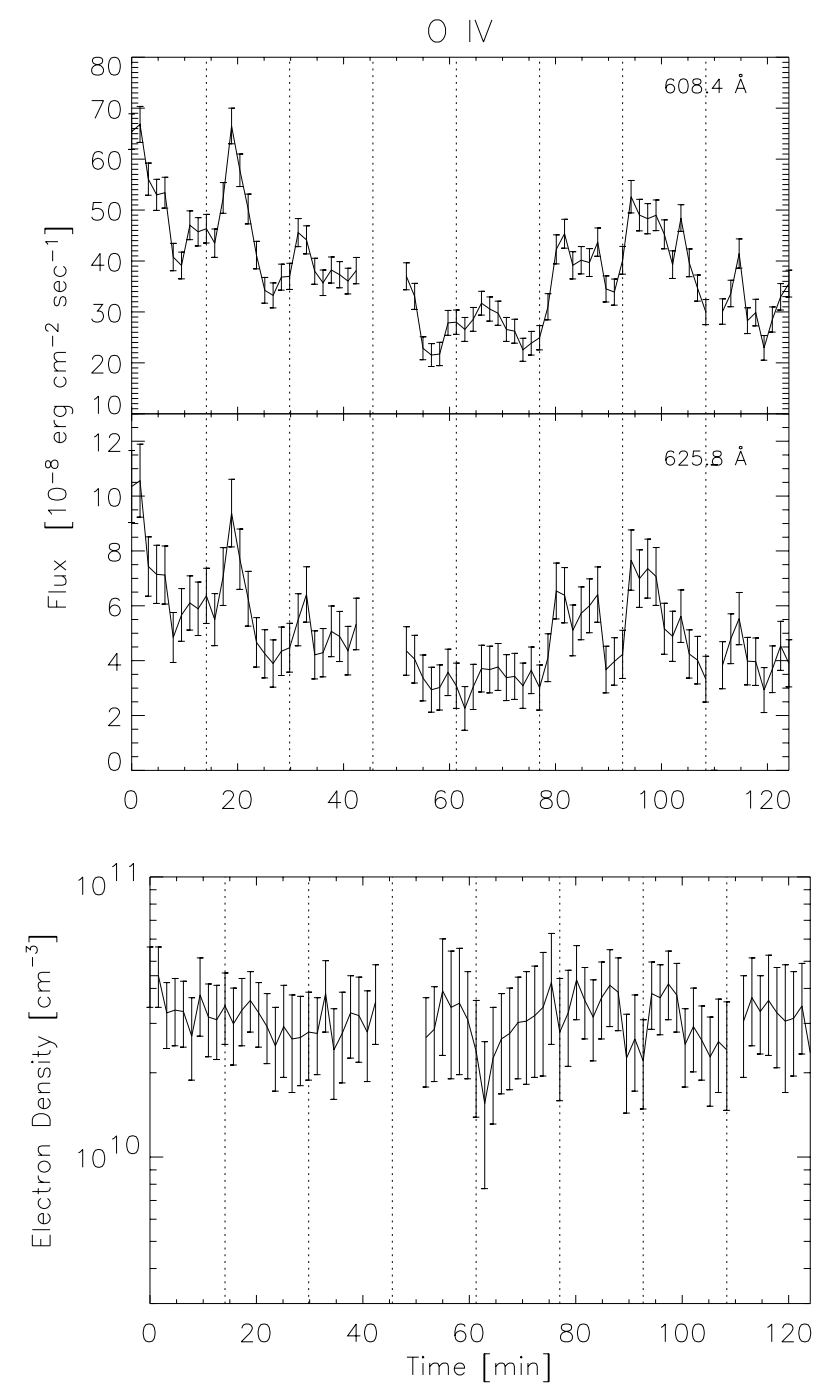

Fig. 12. Top figure: intensity changes along time for the two O IV lines that form the electron density sensitive ratio 625.8/608.4. Bottom figure: electron density changes along time. The start time is the same as in Fig. 7. The dotted lines serve as reference for comparison.

ratio sensitive to electron density changes at this temperature to confirm the result.

\section{Discussion and conclusions}

The association of coronal bright points with bipolar magnetic features was established in the first studies carried on BPs (Krieger et al. 1971; Golub et al. 1977). These works were followed by many more concerning different aspects of the magnetic properties of BPs including statistical (Webb et al. 1993) and individualized (Brown et al. 2001; Madjarska et al. 2003) studies of the evolution of the photospheric magnetic features, the analysis of the solar cycle dependence of the polarities (Sattarov et al. 2002), its orientation with respect to the coronal emission (Longcope et al. 2001) or the correlation with the X-ray and EUV emission (Preś \& Phillips 1999). We know from the latter that the magnetic flux determined from the photospheric magnetic features is well time correlated with the Fe XII coronal emission and the X-ray flux, in the way 
shown in the top and middle panels of our Fig. 5. Our contribution extends the sample to two more BPs, adding the result that not only does the coronal emission rise due to an increase in emission area (which increases too), but also because the BP emits more per unit area as the magnetic flux becomes stronger. Therefore, there is more intrinsic emission when there is more magnetic flux involved. In fact, we find there is a one to one relation between the magnetic flux in the bipolarity and the EIT Fe XII coronal emission for the two BPs, both in the growing and decaying phase. The exception is the brightest moments of the first BP when the high increase in emission is not accompanied by a comparable increase in the magnetic flux. The reason for that is not clear at the moment and more BPs have to be studied to see if there is a saturation limit in the magnetic strength.

These results give further support to the picture that shows a $\mathrm{BP}$ whose emission is a result of the interaction of the magnetic polarities, with most likely magnetic reconnection involved in the process. The converging flux model proposed by Priest et al. (1994) describes how the approach of two opposite polarities creates a $\mathrm{X}$-point that rises into the corona and produces a X-ray bright point (X-ray emission) by coronal reconnection (see also Parnell et al. 1994; Longcope 1998). The model proposes a three phase evolution: preinteraction (approach), interaction and finally cancellation. Several observational questions are raised concerning the evolution of the features. For example, the timing between coronal emission and magnetic cancellation. In this case, BP1 fades (Fe XII $195 \AA$ emission below the threshold) when, after the interaction and almost full cancellation of the positive polarity, the remaining fractions of opposite polarity are $\sim 10 \mathrm{Mm}$ apart. BP2 appears when new positive flux emerges at a distance of $\sim 6 \mathrm{Mm}$ and finally disappears $\sim 3-4 \mathrm{~h}$ before the full cancellation of the positive polarity. This is in agreement with Harvey et al. (1999) who observed several examples of coronal emission disappearance before the cancellation of magnetic network elements and suggested that magnetic flux is submerging at most of these cancellation sites. It would be interesting to check with new observations the timing of disappearance of the emission at different temperatures.

Another key aspect of the evolution of BPs is their high variability in EUV lines and X-rays. In a set of several papers and looking at spectroheliograms obtained from Skylab experiments, Habbal and collaborators (Habbal \& Withbroe 1981; Habbal et al. 1990; Habbal \& Grace 1991) analyzed the intensity fluctuations of several chromospheric (Ly $\alpha, \mathrm{C}$ II), transition region (C III, O IV, O VI) and coronal ( $\mathrm{Mg} \mathrm{X}$ ) lines. They reported significant temporal fluctuations between scans, finding short-term variations of the order of $\sim 5 \mathrm{~min}$, but also more gradual ones (20-30 $\mathrm{min}$ ). These fluctuations were correlated between transition region lines, but not always with variations at other temperatures. The variability was most enhanced at $10^{5} \mathrm{~K}$ and no characteristic periodicities were found. They also pointed out that both quiet Sun and coronal hole bright points behave similarly, concluding that the BP properties are independent of the structure of the overlying large-scale magnetic field. The main limitation of the studies was the temporal resolution of their data, the 5.5 min needed to obtain the spectroheliogram. Our observations with an improved temporal resolution (94 and $36 \mathrm{~s}$ ) have confirmed some of these results. We have found variations in the flux of He I $583.33 \AA$ and O v $629.73 \AA$ with a characteristic response time ranging between 420-650 s. Many of these events appear in a random fashion and sometimes after periods of quietness. The strongest variability is in the transition region lines, well correlated between them, with no counterpart in the changes of the Mg IX 368.07 $\AA$ emission. The coronal emission for this BP seems to evolve following more gradual changes.

The interpretation of the behaviour of He I $583.33 \AA$ is more difficult. It is under debate how the helium lines are formed in the Sun and several mechanisms have been proposed over the years. Which process is the dominant could determine where to expect helium to emit. If photoionization from coronal radiation followed by recombination is dominant, it could be formed in the upper chromosphere, while if that mechanism is not the dominant one, the formation could take place at the lower transition region (Andretta et al. 2003, and references therein). This last scenario would explain why the He I series follow so closely the trend given by the transition region lines and why it does not follow the coronal one. However, to discuss the He lines formation is not the purpose of this paper.

Finally, what it is important to stand out from our observations is the oscillatory behaviour present in the two bright points to which we applied the wavelet analysis. In the SUMER BP, looking at the S VI $933.40 \AA$ intensity fluctuations we have found a peak in the wavelet spectrum over a $95 \%$ significance level at $491 \mathrm{~s}$ during at least four cycles. The sit-andstare mode was used and new plasma was seen under the slit every $\sim 400 \mathrm{~s}$. The oscillatory pattern could be then explained by four-five small structures $\left(\leqslant 1^{\prime \prime}\right)$ evenly spaced, which moved under the slit producing the periodic intensity fluctuations as they pass. However, it seems to us much more likely to have a $4-5^{\prime \prime}$ structure crossing the slit while it experiences oscillations in its emission. Similarly we have found oscillations in the emission of the O v $629.73 \AA$ line of the CDS bright point. The wavelet power spectrum has its peak at $546 \mathrm{~s}$. In this case the oscillation decreases in amplitude producing a damped oscillatory profile with a change in the period in the last few cycles.

The wavelet analysis also provides periods of 900-1000 s for both SUMER and CDS suggesting that there could be a longer modulation component. In the case of the SUMER observations this modulation is only two cycles long. Since new plasma is seen under the slit every $400 \mathrm{~s}$ it could be that this is just a response to the morphology of the bright point. These uncertainties together with the realization that the fine structure of the bright point can only be "resolved" with the highest spatial resolution images, as shown from the comparison of EIT and TRACE images, suggests that coordinated studies of high spatial and temporal resolution images should go hand in hand with high cadence spectroscopic studies, if one wishes to understand better the nature of the oscillations. This issue will be adressed in a forthcoming paper as well as an extended study looking for oscillations at higher temperatures, which have already been found in other coronal features (Aschwanden et al. 1999; O'Shea et al. 2001, and references therein). 
Acknowledgements. Research at Armagh Observatory is grant-aided by the N. Ireland Dept. of Culture, Arts and Leisure. This work was supported in part by PPARC grant PPA/G/S/1999/00055 and PPA/V/S/1999/00628. CDS, EIT, MDI and SUMER are instruments onboard SOHO. SOHO is a project of international cooperation between ESA and NASA. CHIANTI is a collaborative project involving the NRL (USA), RAL (UK), and the Universities of Florence (Italy) and Cambridge (UK).

\section{References}

Andretta, V., Del Zanna, G., \& Jordan, S. D. 2003, A\&A, 400, 737

Aschwanden, M. J., Fletcher, L., Schrijver, C. J., \& Alexander, D. 1999, ApJ, 520, 880

Brooks, D. H., Fischbacher, G. A., Fludra, A., et al. 1999, A\&A, 347, 277

Brown, D. S., Parnell, C. E., Deluca, E. E., Golub, L., \& McMullen, R. A. 2001, Sol. Phys., 201, 305

Chae, J., Wang, H., Qiu, J., et al. 2001, ApJ, 560, 476

Del Zanna, G. 1999, Ph.D. Thesis

Del Zanna, G., Bromage, B. J. I., Landi, E., \& Landini, M. 2001, A\&A, 379, 708

Del Zanna, G., Bromage, B. J. I., \& Mason, H. E. 2003, A\&A, 398, 743

Delaboudiniere, J.-P., Artzner, G. E., Brunaud, J., et al. 1995, Sol. Phys., 162, 291

Dere, K. P., Landi, E., Mason, H. E., Monsignori Fossi, B. C., \& Young, P. R. 1997, A\&AS, 125, 149

Egamberdiev, S. A. 1983, SvA Lett., 9, 385

Fludra, A. 2001, A\&A, 368, 639

Frank, Z. A., \& Slater, G. L. 2002, Am. Astron. Soc. Meet., 201, 0

Gallagher, P. T., Phillips, K. J. H., Harra-Murnion, L. K., \& Keenan, F. P. 1998, A\&A, 335, 733

Golub, L., Krieger, A. S., Harvey, J. W., \& Vaiana, G. S. 1977, Sol. Phys., 53, 111

Golub, L., Krieger, A. S., Silk, J. K., Timothy, A. F., \& Vaiana, G. S. 1974, ApJ, 189, L93

Habbal, S. R., \& Grace, E. 1991, ApJ, 382, 667

Habbal, S. R., \& Withbroe, G. L. 1981, Sol. Phys., 69, 77

Habbal, S. R., Withbroe, G. L., \& Dowdy, J. F. 1990, ApJ, 352, 333
Hagenaar, H. J. 2001, ApJ, 555, 448

Harrison, R. A., Sawyer, E. C., Carter, M. K., et al. 1995, Sol. Phys., 162,233

Harvey, K. L. 1985, Aust. J. Phys., 38, 875

Harvey, K. L. 1993, Ph.D. Thesis

Harvey, K. L., Jones, H. P., Schrijver, C. J., \& Penn, M. J. 1999, Sol. Phys., 190, 35

Howard, R. F., Harvey, J. W., \& Forgach, S. 1990, Sol. Phys., 130, 295

Krieger, A. S., Vaiana, G. S., \& van Speybroeck, L. P. 1971, in Solar Magnetic Fields, IAU Symp., 43, 397

Lau, K. M., \& Weng, H. 1995, Bull. Amer. Meteor. Soc., 76, 2391

Longcope, D. W. 1998, ApJ, 507, 433

Longcope, D. W., Kankelborg, C. C., Nelson, J. L., \& Pevtsov, A. A. 2001, ApJ, 553, 429

Madjarska, M. S., Doyle, J. G., Teriaca, L., \& Banerjee, D. 2003, A\&A, 398, 775

Marsh, K. A., Hurford, G. J., \& Zirin, H. 1980, ApJ, 236, 1017

Mazzotta, P., Mazzitelli, G., Colafrancesco, S., \& Vittorio, N. 1998, A\&AS, 133, 403

Nolte, J. T., Solodyna, C. V., \& Gerassimenko, M. 1979, Sol. Phys., 63,113

O'Shea, E., Banerjee, D., Doyle, J. G., Fleck, B., \& Murtagh, F. 2001, A\&A, 368, 1095

Parnell, C. E., Priest, E. R., \& Titov, V. S. 1994, Sol. Phys., 153, 217

Pauluhn, A., Rüedi, I., Solanki, S. K., et al. 1999, Appl. Opt., 38, 7035

Pike, C. D. 1997, CDS Software Note No. 45

Preś , P., \& Phillips, K. 1999, ApJ, 510, L73

Priest, E. R., Parnell, C. E., \& Martin, S. F. 1994, ApJ, 427, 459

Sattarov, I., Pevtsov, A. A., Hojaev, A. S., \& Sherdonov, C. T. 2002, ApJ, 564, 1042,

Scherrer, P. H., Bogart, R. S., Bush, R. I., et al. 1995, Sol. Phys., 162, 129

Sheeley, N. R., \& Golub, L. 1979, Sol. Phys., 63, 119

Thompson, W. T. 1999, CDS Software Note No. 53

Torrence, C., \& Compo, G. P. 1998, Bull. Amer. Meteor. Soc., 79, 61

Vaiana, G. S., Krieger, A. S., van Speybroeck, L. P., \& Zehnpfennig, T. 1970, Bull. Am. Phys. Soc., 15, 611

Webb, D. F., Martin, S. F., Moses, D., \& Harvey, J. W. 1993, Sol. Phys., 144, 15

Young, P. R., Del Zanna, G., Landi, E., et al. 2003, ApJS, 144, 135

Zhang, J., Kundu, M. R., \& White, S. M. 2001, Sol. Phys., 198, 347 